\title{
Combined pulmonary fibrosis and emphysema characteristics in a Greek cohort
}

\author{
Foteini Malli ${ }^{1}$, Despoina Papakosta ${ }^{2}$, Katerina Antoniou ${ }^{3}$, Maria Dimadi ${ }^{4}$, \\ Vlassis Polychronopoulos ${ }^{5}$, Katerina Malagari ${ }^{6}$, Anastasia Oikonomou$^{7}$, \\ Demosthenes E. Bouros ${ }^{8}$ and Zoe Daniil ${ }^{1}$
}

\begin{abstract}
Affiliations: ${ }^{1}$ Respiratory Medicine Dept, University of Thessaly, School of Medicine, Larissa, Greece. ${ }^{2}$ University of Thessaloniki, G. Papanikolaou Hospital, Dept of Pneumonology Thessaloniki, Thessaloniki, Greece. ${ }^{3}$ Dept of Thoracic Medicine and Laboratory of Molecular and Cellular Pneumonology, Interstitial Lung Disease Unit, University Hospital of Heraklion, Heraklion, Greece. " "SOTIRIA" Athens Chest Hospital, 1st Dept of Pneumonology, Athens, Greece. ${ }^{5}$ Ygeia Hospital Athens, Athens, Greece. ${ }^{6}$ Evgenidion University Hospital, Imaging and Research Unit, Athens, Greece. ${ }^{7}$ Democritus University of Thrace, Radiology Dept, Alexandroupolis, Greece. ${ }^{8}$ Interstitial Lung Diseases Unit, Hospital for Diseases of the Chest Sotiria, National and Kapodistrian University of Athens, Medical School, Athens, Greece.
\end{abstract}

Correspondence: Katerina Antoniou, Faculty of Medicine, University of Crete, Dept of Thoracic Medicine, Heraklion 71110 Crete, Greece. E-mail: katerinaantonioudyahoo.gr

\section{ABSTRACT}

Background: Combined pulmonary fibrosis and emphysema (CPFE) has recently received great attention, with studies suggesting that it presents a distinct clinical entity while others have challenged this hypothesis. This nationwide study aimed to describe a large cohort of Greek CPFE patients and to examine potential prognostic factors for survival.

Methods: This retrospective study included 97 patients with CPFE. Demographic and clinical data, pulmonary function tests, echocardiography results and bronchoalveolar lavage analysis were recorded.

Results: Most patients were male (94.8\%) and 92\% were current or ex-smokers. Spirometry results were abnormal (forced vital capacity (FVC) $72.9 \pm 19.9 \%$ pred and forced expiratory volume in $1 \mathrm{~s} / \mathrm{FVC}$ $82.9 \pm 9.7 \%)$ with reduced diffusing capacity of the lung for carbon monoxide (DLCO) $(42.3 \pm 17.4 \%$ pred). Mean systolic pulmonary arterial pressure was $41.9 \pm 19.7 \mathrm{mmHg}$ and pulmonary hypertension was present in $58.8 \%$ of patients. Mean 6-min walk distance was $335.4 \pm 159.4 \mathrm{~m}$. Mean emphysema score was $14.23 \pm 8.69 \%$ and mean interstitial lung disease (ILD) extent was $39.58 \pm 19.82 \%$. Mean survival was 84 months (95\% CI 72-96 months). Patients with DLCO $\geqslant 39 \%$ pred had better survival than patients with DLCO $<39 \%$ pred $(p=0.031)$. Patients with ILD extent $\geqslant 30 \%$ had worse survival than patients with ILD extent $<30 \%(\mathrm{p}=0.037)$.

Conclusions: Our results indicate that CPFE patients have preserved lung volumes associated with disproportionately reduced DLCO, while reduced DLCO and increased ILD extent was associated with worse prognosis.

@ERSpublications

Prognosis of CPFE is associated with pulmonary function status and ILD extent http://ow.ly/izvd30nHFgh

Cite this article as: Malli F, Papakosta D, Antoniou K, et al. Combined pulmonary fibrosis and emphysema characteristics in a Greek cohort. ERJ Open Res 2019; 5: 00014-2018 [https://doi.org/ 10.1183/23120541.00014-2018].

This article has supplementary material available from openres.ersjournals.com.

Received: Jan 292018 | Accepted after revision: Jan 252019

Copyright $\odot$ ERS 2019. This article is open access and distributed under the terms of the Creative Commons Attribution Non-Commercial Licence 4.0. 


\section{Introduction}

Combined emphysema with pulmonary fibrosis (CPFE) has recently gathered increasing interest, and researchers have proposed that CPFE may represent a distinct clinical syndrome with specific clinical, radiological and functional characteristics, along with a different prognosis $[1,2]$. According to previous studies, CPFE patients have preserved lung volumes with greatly reduced diffusing capacity of the lung for carbon monoxide (DLCO). Clinicians have suggested that CPFE is a distinct clinical syndrome, while the recently published guidelines concerning the classification of idiopathic interstitial pneumonias have not included CPFE as an individual entity but have described it as a coexisting pattern of emphysema with chronic fibrosing interstitial pneumonias, underlining the need for further investigations [3]. Additionally, CPFE is associated with a high prevalence of pulmonary hypertension [4]. In terms of prognosis, the data in the literature are rather contradictory. Some have reported worse outcome in CPFE patients [5] while others have observed better prognosis when compared to idiopathic pulmonary fibrosis (IPF) without emphysema [6].

The objective of the present study was to provide a detailed description of the characteristics of CPFE in a Greek cohort and to determine factors that may influence survival.

\section{Methods}

\section{Study design}

This retrospective, multicentre study was conducted by the Hellenic Interstitial Lung Diseases Group. Patients presenting to Greek referral centres and evaluated by physicians with a special interest in interstitial lung diseases (ILDs) were included in this study between March 2011 and December 2014. Patients were recruited by seven Greek ILD centres, namely the Respiratory Medicine Dept of the University of Thessaly (University Hospital of Larissa; 16 patients), the Dept of Pneumonology of the University of Thessaloniki (G. Papanikolaou Hospital; 13 patients), the Dept of Thoracic Medicine and Interstitial Lung Disease Unit of the University Hospital of Heraklion (18 patients), the 1st Dept of Pneumonology of "SOTIRIA" Athens Chest Hospital (National and Kapodistrian University of Athens; 27 patients), the Respiratory Medicine Dept of the "Sismanoglion" General District Hospital (Athens; five patients) and the Dept of Pneumonology of the University Hospital of Alexandroupolis (18 patients).

Only patients with a chest high-resolution computed tomography (HRCT) scan available for review were included in the study. The study was approved by the respective ethics committee of each institution. All patients included provided written and verbal consent. Some of these patients were included in a previous study [7].

\section{Study population}

Subjects were included in the study if the following criteria were met: 1) consistent clinical presentation; 2) presence of emphysema on HRCT scan defined as well-demarcated areas of decreased attenuation with very thin wall $(<1 \mathrm{~mm})$ or no wall in the upper zones of the lungs; and 3) presence of HRCT features consistent with definite usual interstitial pneumonia (UIP) such as reticular opacities, honeycombing and/ or traction bronchiectasis with basal and subpleural predominance, and absence of features inconsistent with UIP [8]. In a subgroup of patients who did not fulfil the HRCT criteria for definite UIP, the diagnosis was established by surgical lung biopsy. We chose to include only patients with a firm diagnosis of IPF in order to avoid bias due to inclusion of patients with other type of ILDs with different prognosis and, most of the time, better prognosis than IPF. Demographic and clinical data were obtained from all participants, including symptoms, smoking status, pulmonary function tests (PFTs), 6-min walk test results, arterial blood gas analysis, serology for autoantibodies and bronchoalveolar lavage fluid analysis. The use of right heart catherization, which is the gold standard for the diagnosis of pulmonary hypertension for research purposes alone, is associated with poor patient consent and, therefore, the presence of pulmonary hypertension was assessed by transthoracic echocardiogram (which presents a noninvasive and easily applicable method). Pulmonary hypertension was defined as systolic pulmonary arterial pressure (sPAP) $\geqslant 35 \mathrm{mmHg}$ [9-11]. sPAP was estimated as previously reported [11]. Linear internal measurements of the left ventricle and its walls were performed in the parasternal long-axis view while right ventricle dimensions were estimated from a right ventricle-focused apical four-chamber view.

Survival time was assessed as follows: for patients that died during the study period, survival time was calculated from the date of diagnosis to the date of death; for patients that were alive at the end of the study period, survival time was calculated from the date of diagnosis to the date of the end of the study (i.e. December 2014).

\section{Radiological features}

All patients had a HRCT of the chest performed and reviewed by two thoracic radiologists (K. Malagari and A. Oikonomou) who were blinded to clinical and histopathological data. The following features were 
quantified at five levels as previously described [12] (apex to origin of great vessels, main carina, pulmonary venous confluence, between the third and fifth level, and $1 \mathrm{~cm}$ above the hemidiaphragm). The overall extent of ILD (reticular pattern and ground-glass opacification) was estimated to the nearest $5 \%$. The extent of emphysema, defined as well-demarcated areas of low attenuation emarginated by thin wall $(<1 \mathrm{~mm})$ or no wall, was quantified to the nearest $5 \%$. For a more comprehensive analysis of quantification of ILD and emphysema extent, the reader is referred to the supplementary material.

\section{Statistical analysis}

Data are presented as mean \pm SD unless otherwise indicated. Normal distribution was assessed by the Kolmogorov-Smirnov test. A survival curve was determined using the Kaplan-Meier method and was analysed for statistical significance using the log-rank test. Statistical analysis was performed using the SPSS 16 statistical package (SPSS Inc., Chicago, IL, USA). Statistical significance was defined as p<0.05.

\section{Results}

Demographic and clinical characteristics of the participants are shown in table 1. The study population consisted of 97 subjects with a mean \pm SD age of $68.2 \pm 9.0$ years. Of the patients studied, $94.8 \%$ were male. $\sim 92 \%$ of patients were current or ex-smokers while the remaining were lifelong nonsmokers. As far as comorbidities is concerned, $14.43 \%$ of patients presented coronary artery disease, $58.76 \%$ of patients presented arterial hypertension and $25.97 \%$ of patients presented diabetes mellitus. Histological confirmation of the UIP pattern was available in $26.8 \%$ of subjects. Finger clubbing was documented in $63.9 \%$ patients. All patients had dyspnoea and $75.2 \%$ of subjects reported cough. The mean disease duration (estimated from diagnosis until the inclusion of the patient in the study) was $3.5 \pm 2.8$ years. Increased autoantibodies titres (antinuclear antibodies and/or rheumatoid factor) were present at $9.27 \%$ of the study population.

The results of the PFT analysis are shown in table 2. Mean values from spirometry indicated a mild restrictive ventilatory defect. Gas exchange was severely impaired as indicated by the reduced DLCO. Lung volumes were moderately reduced (table 2). Mean arterial oxygen tension at rest was reduced and as expected, mean alveolar-arterial oxygen tension gradient was increased (table 2). Data from the transthoracic echocardiogram were available in 68 patients. Mean sPAP was $41.9 \pm 19.7 \mathrm{mmHg}$ and pulmonary hypertension was present in $58.8 \%$ of the study participants. Mean left ventricle ejection fraction was $62.02 \pm 9.44 \%$, mean left ventricular end-systolic dimension was $32.80 \pm 7.83 \mathrm{~mm}$, mean left ventricular end-diastolic dimension was $36.23 \pm 7.89 \mathrm{~mm}$ and mean right ventricle basal dimeter was $36.57 \pm 6.45 \mathrm{~mm}$. Mean 6-min walk distance was $335.4 \pm 159.4 \mathrm{~m}$.

Bronchoalveolar lavage fluid analysis was available in $51.5 \%$ of the participants. The mean total cell count was $17.7 \pm 14.0 \times 10^{5}$ per $\mathrm{mL}$. The differential cell counts are shown in table 3 . Mean emphysema score was $14.2 \pm 8.6 \%$ and mean ILD extent was $39.6 \pm 19.8 \%$.

\section{TABLE 1 Demographic characteristics and clinical manifestations of the study population}

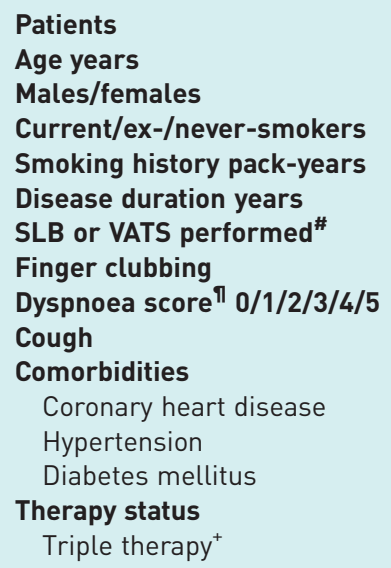

Data are presented as mean \pm SD, unless otherwise stated. SLB: surgical lung biopsy; VATS: video-assisted thoracoscopic surgery. "\#: usual interstitial pneumonia pattern; ": Medical Research Council scale; ${ }^{+}$: combination of $\mathrm{N}$-acetylcysteine, steroids and azathioprine. 
TABLE 2 Pulmonary function tests, arterial blood gas analysis and systolic pulmonary arterial pressure (SPAP) data of the study population

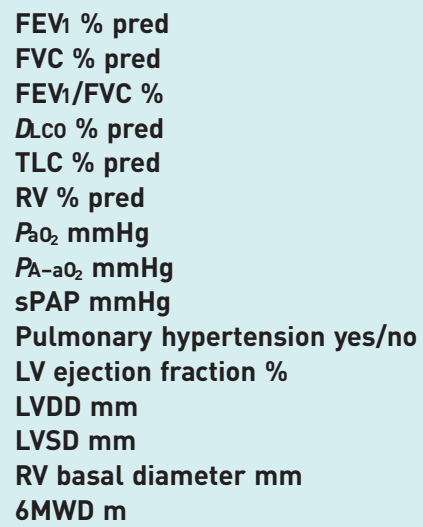

$75.76 \pm 20.60$

$72.92 \pm 19.95$

$82.90 \pm 9.73$

$42.35 \pm 17.38$

$66.43 \pm 16.80$

$64.18 \pm 22.08$

$68.28 \pm 11.31$

$36.66 \pm 19.39$

$41.92 \pm 19.69$

Data are presented as mean $\pm \mathrm{SD}$, unless otherwise stated. $\mathrm{FEV} 1$ : forced expiratory volume in $1 \mathrm{~s}$; FVC: forced vital capacity; DLCO: diffusing capacity of the lung for carbon monoxide; TLC: total lung capacity; RV: residual volume; $\mathrm{PaO}_{2}$ : arterial oxygen tension; $P_{\mathrm{A}-\mathrm{aO}_{2}}$ : aveolar-arterial oxygen tension gradient; LV: left ventricular; DD: end-diastolic dimension; SD: end-systolic dimension; RV: right ventricular; 6MWD: 6-min walking distance.

\section{Survival analysis}

Mean survival was 84 months (95\% CI 72-96 months) after the diagnosis. We stratified the patient group according to the presence or absence of pulmonary hypertension as assessed by Doppler echocardiography using the threshold of sPAP $35 \mathrm{mmHg}$. We observed that patients with sPAP $\geqslant 35 \mathrm{mmHg}$ did not differ in terms of prognosis with patients with $\mathrm{sPAP}<35 \mathrm{mmHg}$ (figure 1). We additionally stratified patients according to values of sPAP (i.e. sPAP $<30,31-40,41-50$ and $>51 \mathrm{mmHg}$ ) and did not observe differences in prognosis (data not shown). Additionally, we stratified patients according to disease severity (as assessed by $D$ LCO $\%$ pred) as mild to moderate ( $D$ LCO $\geqslant 39 \%$ pred) or severe ( $D$ LCO $<39 \%$ pred). We observed that patients with $D$ LCO $\geqslant 39 \%$ pred had better survival than patients with severe disease ( $p=0.031$ ) (figure 2). Interestingly, patients with ILD extent $\geqslant 30 \%$ presented worse prognosis than those with ILD extent $<30 \%$ $(\mathrm{p}=0.037$ ) (figure 3 ). The presence of comorbidities (i.e. coronary artery disease, hypertension or diabetes mellitus) or therapy status were not determinants of prognosis (data not shown).

\section{Discussion}

In this multicentre study, we observed that CPFE subjects presented a mild restrictive spirometry associated with reduced gas exchange. Moreover, reduced DLCO and increased ILD extent were associated with poor prognosis. Mean survival in our population was 84 months. Notably, the majority of our patients presented signs of vascular involvement as sPAP was $41.92 \mathrm{mmHg}$. In this respect, our observations give further insight in the clinical course of the CPFE population.

\section{TABLE 3 Bronchoalveolar lavage (BAL) fluid analysis and high-resolution computed} tomography (HRCT) findings

BAL performed yes/no

Data are presented as mean $\pm \mathrm{SD}$, unless otherwise stated. ILD: interstitial lung disease. 
FIGURE 1 Kaplan-Meier curve of combined pulmonary fibrosis and emphysema patients stratified by the presence or absence of pulmonary hypertension as assessed by echocardiography li.e. systolic pulmonary arterial pressure $\geqslant 35 \mathrm{mmHg}$.

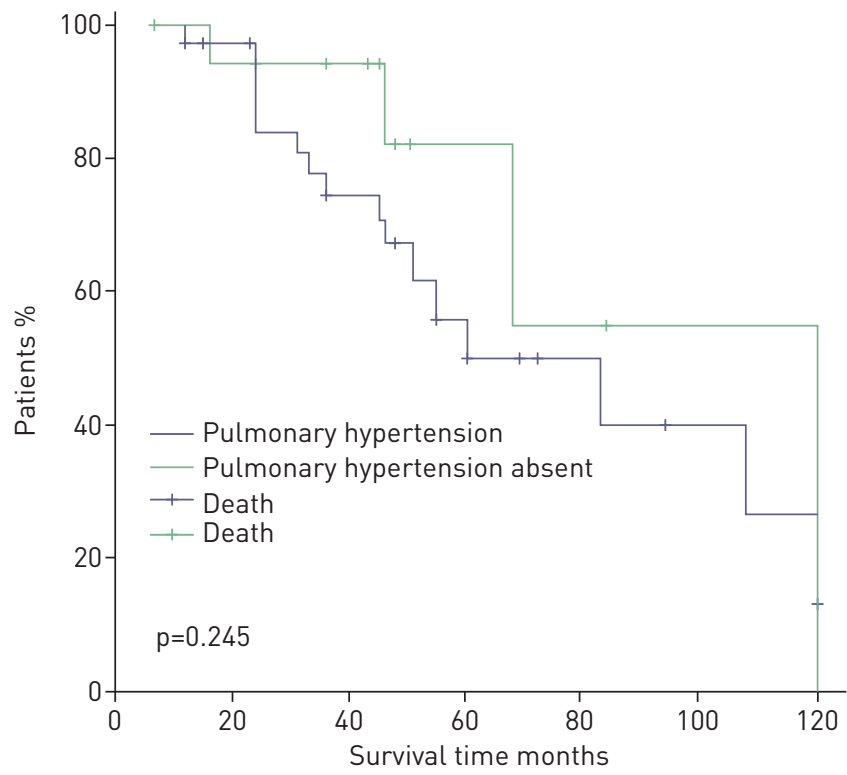

We observed that CPFE patients in our cohort presented a rather subnormal spirometry with reduced DLCO possibly due to the additive effects of the two diseases in DLCO and their opposing effects in elastic recoil [4]. Our results are in accordance with previously published data indicating that spirometry values, and mainly FVC alone, cannot be used in order to screen or to monitor patients with CPFE and do not allow an accurate assessment of prognosis. Additionally, we observed that static lung volumes were reduced with a mean total lung capacity of $66.43 \pm 16.80 \%$ pred, indicating a predominantly restrictive pattern. Previous published data have reported normal static lung volumes in CPFE patients [13], while others have demonstrated a mild reduction. Tond et al. [6] have reported that lung volumes vary according to the extent of emphysema and thus may explain the discrepancy among the studies.

The natural history of CPFE is largely unknown. We show that the mean survival in our cohort was 84 months following diagnosis. We chose to include only patients with an IPF pattern in order to avoid bias due to inclusion of patients with other type sof ILDs with different prognoses to IPF. We did not include a control group of IPF subjects; therefore, we cannot directly compare the CPFE survival with that of IPF. However, according to previously published data, it seems that CPFE subjects carry equal or even better prognosis versus IPF alone [6,13-16] while others have challenged this conclusion [17, 18]. The reason(s) for these differences in survival of CPFE compared to IPF are not clear but one could argue that

FIGURE 2 Kaplan-Meier curve of combined pulmonary fibrosis and emphysema patients stratified by diffusing capacity of the lung for carbon monoxide (DLCO) \% pred.

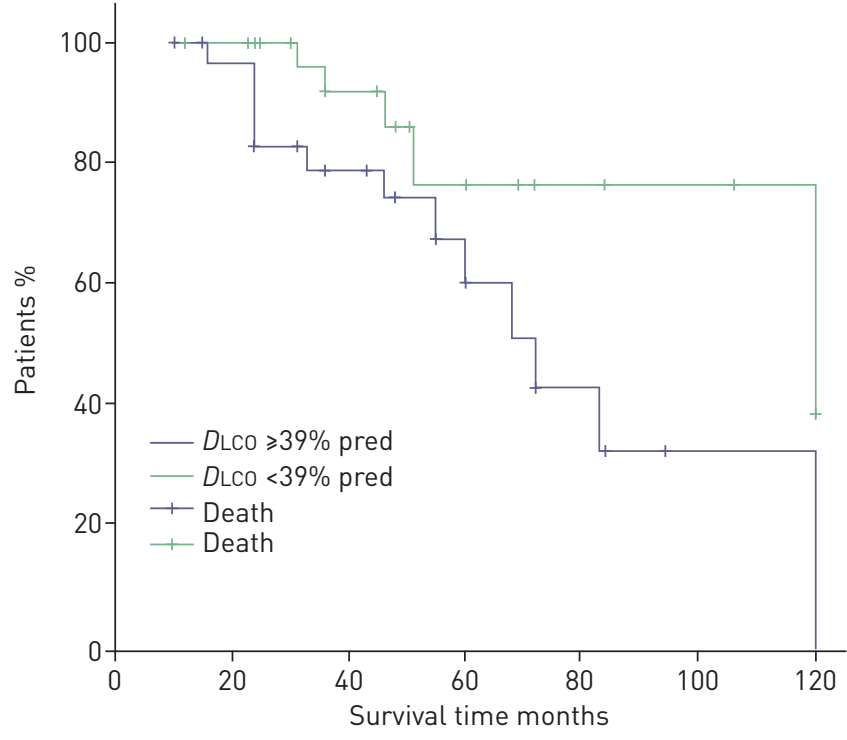


FIGURE 3 Kaplan-Meier curve of combined pulmonary fibrosis and emphysema patients stratified by interstitial lung disease (ILD) extent.

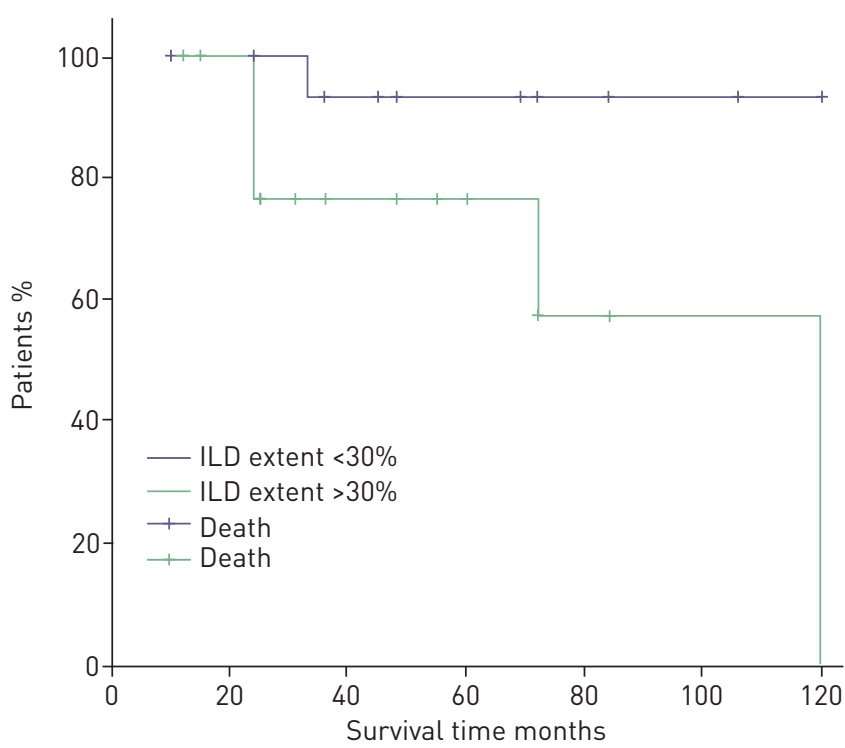

this may be the result of the overlapping pathophysiological mechanisms implicated in the two entities, resulting in different clinical course of IPF or emphysema alone [18, 19]. Another possible explanation could be differences in the prevalence of comorbidities such as lung cancer [20]. Differences in metalloproteinase expression [21], surfactant protein $C$ gene mutations [22], overexpression of tumour necrosis factor- $\alpha$ [23] and platelet-derived growth factor-B [24] in animal models have been implicated, among other factors, in the development of CPFE. Additionally, the discordance among the studies concerning CPFE versus IPF survival may be due to different definitions of CPFE among studies. The present study was not designed to address this hypothesis that needs further investigation in future studies.

Studies have previously shown that decline in DLCO is associated with worse prognosis in CPFE subjects [25]. Our results are in accordance with previous findings and indicate that DLCO may serve as a predictor of mortality in the CPFE population. We demonstrated that DLCO $<39 \%$ pred is associated with worse prognosis when compared to patients with DLCO $\geqslant 39 \%$ pred. Some have suggested that DLCO may correlate with the degree of severity and parenchymal destruction better than other physiological parameters like FVC due to the opposing effects of the two diseases in elastic recoil [26, 27]. However, it should be stressed that DLCO may be influenced by the presence of pulmonary vascular involvement. Interestingly, others have suggested that a decline in forced expiratory volume in $1 \mathrm{~s}$ may be the best marker for predicting mortality in CPFE subjects while DLCO may not accurately identify patients at risk [26].

The association of CPFE with pulmonary hypertension has received significant attention in recent years. Pulmonary hypertension is highly prevalent in CPFE patients and in agreement with earlier studies [2, 4], we demonstrated that $>50 \%$ of our patient population probably presented pulmonary hypertension, with a mean sPAP of $41.9 \pm 19.7 \mathrm{mmHg}$. The presence of pulmonary hypertension has previously been recognised as a negative predictor of mortality in CPFE patients. However, we did not observe any differences in survival in patients with and without pulmonary hypertension. One possible explanation for the discrepancy is the use of transthoracic echocardiography to diagnose pulmonary hypertension and/or the lack of a consensus definition of CPFE in the literature. We have included only subjects with a UIP pattern either in HRCT or lung tissue biopsy, while others have used less strict criteria, thus including patients with a nonspecific interstitial pneumonia pattern in the analyses [28].

In the present study, we observed that increased ILD extent may have a negative impact on prognosis. Others have challenged this finding by reporting no influence of pulmonary fibrosis score on survival [18] while ЈАСОВ et al. [16] reported that the total extent of fibrosis and emphysema predicts mortality in CPFE patients. Our observations suggest that CPFE outcome may be worsened by the IPF component of the disease. ILD extent may reflect overall severity of lung parenchyma involvement and through that mechanism, may be implicated in CPFE survival. Additionally, we did not observe any association of therapy status with survival. At the time the study was performed, the only available therapy in Greece was the triple combination of azathioprine, prednisolone and $\mathrm{N}$-acetylcysteine. The small number of patients receiving therapy in our cohort renders major uncertainty to the latter finding. 
Our study has several limitations. One major criticism of our findings is the lack of right heart catheterisation as a more accurate measure of pulmonary haemodynamics and as a means of assessing the presence of pulmonary hypertension, which may be implicated in the diversity of our results with those previously published [4]. We acknowledge that Doppler echocardiography may be misleading in the assessment of patients with suspected pulmonary hypertension and may not adequately identify pulmonary hypertension, especially in the setting of lung disease [29]. Right heart catheterisation, which is a rather invasive method, is the gold standard for the diagnosis of pulmonary hypertension. However, echocardiography serves as a noninvasive and easily applicable method that is better tolerated by the patient for research purposes alone and, therefore, is associated with better patient consent. Taking this into account, we acknowledge that based on our findings, no definite conclusions can be drawn concerning the prevalence and prognostic ability of pulmonary hypertension in patients with CPFE. Additionally, our study has an observational and retrospective design, and therefore our patients may be subject to selection bias. Another limitation is the lack of serial PFT data that might provide a helpful predictor of prognosis. Finally, we acknowledge that to have a complete evaluation of survival analysis, patients with CPFE need to be compared with a matched group of IPF patients and a second group of emphysema patients.

\section{Conclusions}

In conclusion, we have observed that patients with CPFE present preserved lung volumes associated with disproportionately reduced DLCO. Reduced DLCO and increased ILD extent was associated with worse prognosis. Further clarifications are warranted to clarify the clinical course and the pathophysiological mechanisms involved in CPFE.

Acknowledgements: We acknowledge the contribution of the following colleagues, who made this report possible. K. Manika (Aristotle University of Thessaloniki G. Papanikolaou Hospital, Thessaloniki, Greece), F. Bardaka (University of Thessaly, Larissa, Greece), G.A. Margaritopoulos (University Hospital of Heraklion, Heraklion, Greece), A. Tzouvelekis ("SOTIRIA" Athens Chest Hospital, 1st Dept of Pneumonology, Athens, Greece) and F. Drakopanagiotakis ("Sismanoglion" General District Hospital, Athens, Greece).

Conflict of interest: None declared.

\section{References}

1 Cottin V, Cordier JF. The syndrome of combined pulmonary fibrosis and emphysema. Chest 2009; 136: 1-2.

2 Cottin V, Nunes H, Brillet PY, et al. Combined pulmonary fibrosis and emphysema: a distinct underrecognised entity. Eur Respir J 2005; 26: 586-593.

3 Travis WD, Costabel U, Hansell DM, et al. An official American Thoracic Society/European Respiratory Society statement: update of the international multidisciplinary classification of the idiopathic interstitial pneumonias. Am J Respir Crit Care Med 2013; 188: 733-748.

4 Cottin V, Le Pavec J, Prevot G, et al. Pulmonary hypertension in patients with combined pulmonary fibrosis and emphysema syndrome. Eur Respir J 2010; 35: 105-111.

5 Mejia M, Carrillo G, Rojas-Serrano J, et al. Idiopathic pulmonary fibrosis and emphysema: decreased survival associated with severe pulmonary arterial hypertension. Chest 2009; 136: 10-15.

6 Todd NW, Jeudy J, Lavania S, et al. Centrilobular emphysema combined with pulmonary fibrosis results in improved survival. Fibrogenesis Tissue Repair 2011; 4: 6.

7 Tzouvelekis A, Zacharis G, Oikonomou A, et al. Increased incidence of autoimmune markers in patients with combined pulmonary fibrosis and emphysema. BMC Pulm Med 2013; 13: 31.

8 Raghu G, Collard HR, Egan JJ, et al. An official ATS/ERS/JRS/ALAT statement: idiopathic pulmonary fibrosis: evidence-based guidelines for diagnosis and management. Am J Respir Crit Care Med 2011; 183: 788-824.

9 Barst RJ, McGoon M, Torbicki A, et al. Diagnosis and differential assessment of pulmonary arterial hypertension. J Am Coll Cardiol 2004; 43: Suppl. S, 40S-47S.

10 Joppa P, Petrasova D, Stancak B, et al. Systemic inflammation in patients with COPD and pulmonary hypertension. Chest 2006; 130: 326-333.

11 Rudski LG, Lai WW, Afilalo J, et al. Guidelines for the echocardiographic assessment of the right heart in adults: a report from the American Society of Echocardiography endorsed by the European Association of Echocardiography, a registered branch of the European Society of Cardiology, and the Canadian Society of Echocardiography. J Am Soc Echocardiogr 2010; 23: 685-713.

12 Desai SR, Veeraraghavan S, Hansell DM, et al. CT features of lung disease in patients with systemic sclerosis: comparison with idiopathic pulmonary fibrosis and nonspecific interstitial pneumonia. Radiology 2004; 232 560-567.

13 Jankowich MD, Rounds S. Combined pulmonary fibrosis and emphysema alters physiology but has similar mortality to pulmonary fibrosis without emphysema. Lung 2010; 188: 365-373.

14 Ryerson CJ, Hartman T, Elicker BM, et al. Clinical features and outcomes in combined pulmonary fibrosis and emphysema in idiopathic pulmonary fibrosis. Chest 2013; 144: 234-240.

15 Tasaka S, Mizoguchi K, Funatsu Y, et al. Cytokine profile of bronchoalveolar lavage fluid in patients with combined pulmonary fibrosis and emphysema. Respirology 2012; 17: 814-820.

16 Jacob J, Bartholmai BJ, Rajagopalan S, et al. Functional and prognostic effects when emphysema complicates idiopathic pulmonary fibrosis. Eur Respir J 2017; 50: 1700379.

17 Cottin V, Cordier JF, Wells AU. Centrilobular emphysema combined with pulmonary fibrosis results in improved survival: a response. Fibrogenesis Tissue Repair; 4: 16. 
Zhang L, Zhang C, Dong F, et al. Combined pulmonary fibrosis and emphysema: a retrospective analysis and clinical characteristics, treatment and prognosis. BMC Pulm Med 2016; 16: 137.

19 Lin H, Jiang S. Combined pulmonary fibrosis and emphysema (CPFE): an entity different from emphysema or pulmonary fibrosis alone. J Thorac Dis 2015; 7: 767-779.

20 Sugino K, Ishida F, Kikuchi N, et al. Comparison of clinical characteristics and prognostic factors of combined pulmonary fibrosis and emphysema versus idiopathic pulmonary fibrosis alone. Respirology 2014; 19: $239-245$.

21 Antoniou KM, Hansell DM, Rubens MB, et al. Idiopathic pulmonary fibrosis: outcome in relation to smoking status. Am J Respir Crit Care Med 2008; 177: 190-194.

22 Rogliani P, Mura M, Mattia P, et al. HRCT and histopathological evaluation of fibrosis and tissue destruction in IPF associated with pulmonary emphysema. Respir Med 2008; 102: 1753-1761.

23 Cottin V, Reix P, Khouatra C, et al. Combined pulmonary fibrosis and emphysema syndrome associated with familial SFTPC mutation. Thorax 2011; 66: 918-919.

24 Lundblad LK, Thompson-Figueroa J, Leclair T, et al. Tumor necrosis factor- $\alpha$ overexpression in lung disease: a single cause behind a complex phenotype. Am J Respir Crit Care Med 2005; 171: 1363-1370.

25 Hoyle GW, Li J, Finkelstein JB, et al. Emphysematous lesions, inflammation, and fibrosis in the lungs of transgenic mice overexpressing platelet-derived growth factor. Am J Pathol 1999; 154: 1763-1775.

26 Schmidt SL, Nambiar AM, Tayob N, et al. Pulmonary function measures predict mortality differently in IPF versus combined pulmonary fibrosis and emphysema. Eur Respir J 2011; 38: 176-183.

27 Cottin V, Hansell DM, Sverzellati N, et al. Effect of emphysema extent on serial lung function in patients with idiopathic pulmonary fibrosis. Am J Respir Crit Care Med 2017; 196: 1162-1171.

28 Portillo K, Morera J. Combined pulmonary fibrosis and emphysema syndrome: a new phenotype within the spectrum of smoking-related interstitial lung disease. Pulm Med 2012; 2012: 867870.

29 Keir GJ, Wort SJ, Kokosi M, et al. Pulmonary hypertension in interstitial lung disease: limitations of echocardiography compared to cardiac catheterization. Respirology 2018; 23: 687-694. 